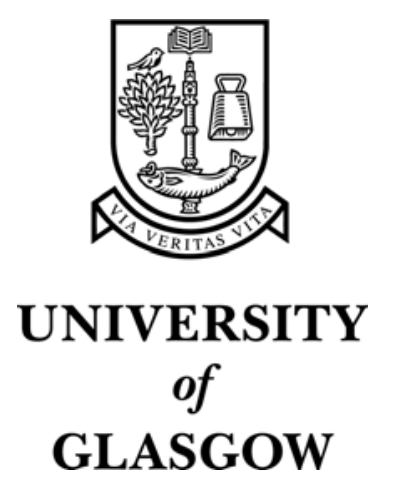

Yu, W. and Kangas, K. (2003) Web-based haptic applications for blind people to create virtual graphs. In, 11th Symposium on Haptic Interfaces for Virtual Environment and Teleoperator Systems, 22-23 March 2003, pages pp. 318-325, Los Angeles, California.

http://eprints.gla.ac.uk/3275/ 


\section{Web-based Haptic Applications for Blind People to Create Virtual Graphs}

\author{
Wai Yu \\ Virtual Engineering Centre, \\ Queen's University of Belfast, \\ Northern Ireland \\ w.yu@qub.ac.uk \\ http://www.vec.qub.ac.uk
}

\author{
Katri Kangas, Stephen Brewster \\ Glasgow Interactive Systems Group, \\ Department of Computing Science, University \\ of Glasgow, U. K. \\ stephen@dcs.gla.ac.uk, \\ http://www.multivis.org
}

\begin{abstract}
Haptic technology has great potentials in many applications. This paper introduces our work on delivery haptic information via the Web. A multimodal tool has been developed to allow blind people to create virtual graphs independently. Multimodal interactions in the process of graph creation and exploration are provided by using a low-cost haptic device, the Logitech WingMan Force Feedback Mouse, and Web audio. The Web-based tool also provides blind people with the convenience of receiving information at home. In this paper, we present the development of the tool and evaluation results. Discussions on the issues related to the design of similar Web-based haptic applications are also given.
\end{abstract}

\section{Introduction}

The aim of the work reported here is to develop a lowcost tool which is not only capable of conveying graphical information to blind people but also allows them to create virtual graphs without the help of a sighted person. The tool is Web-based so that it takes the advantages of the Web such as the popularity, cost-effectiveness and conveniences and provides them to blind people. The types of graphs that blind people can create by using this tool include line graphs, bar charts and pie charts.

We use graphs to present ideas and information more effectively and thus they are useful tools for communication. We usually learned the skills to use them when at school. However the advantages of visualisation tools become less meaningful for blind people. Therefore their access to numerical information is a long and usually sequential process.
To tackle this problem, the traditional approach is to convert visual representations into tactile diagrams so that blind people can feel the general arrangement of graphs through touch. Usually, information is raised on special swell-paper. Through the tactile sensation of their finger tips, blind people can pick up partial information on the raised paper and then assemble a mental image of the graph. This is not an easy task due to the lack of overview of the graph layout moreover the successful use is often affected by the complexity of the graphs and the tactile sensitivity of individuals. Nevertheless, it closely resembles the visual graphs used by sighted people and thus provides a common medium for sighted and blind people to communicate ideas and information.

We have been working on the development of multimodal tools for blind people to access computergenerated graphs. The objective is to overcome the limitations of the existing assistive tools, such as tactile diagrams and audio tablets, by using haptic technology and virtual reality (VR). Success has been found from our evaluations of the multimodal tools with blind people [1]. Based on this success, we have started investigating a new approach, in which tools are being developed to allow blind people to create graphs by themselves. Enabling blind people to draw graphs seems to be less essential than other essential life surviving skills taught at school such as knowing how to cross the road, recognising bank notes, and signing signatures etc. However, graphs are very important for education and work. Karmel and Landay's work has shown that blind people would like to draw and can draw if the technology permits [2]. Moreover, Van Scoy et al have demonstrated a system 
that allows blind students to plot haptic graphs by entering a set of equations [3].

Currently, blind students' choices of study subjects and career paths are very limited compared to their sighted counterparts [3] because scientific subjects often require the usage of graphs and diagrams. To teach blind students graphs, pins, rubber bands and wooden boards are used (Figure 1). Students have to use these tools in order to construct a graph and learn the relationship between data points. However, this technique has limitations such as inaccurate representation of the graphs, possible injury by the sharp pins, and inflexibility to modifications. As a result, blind people tend not to use graphs once they left the school.

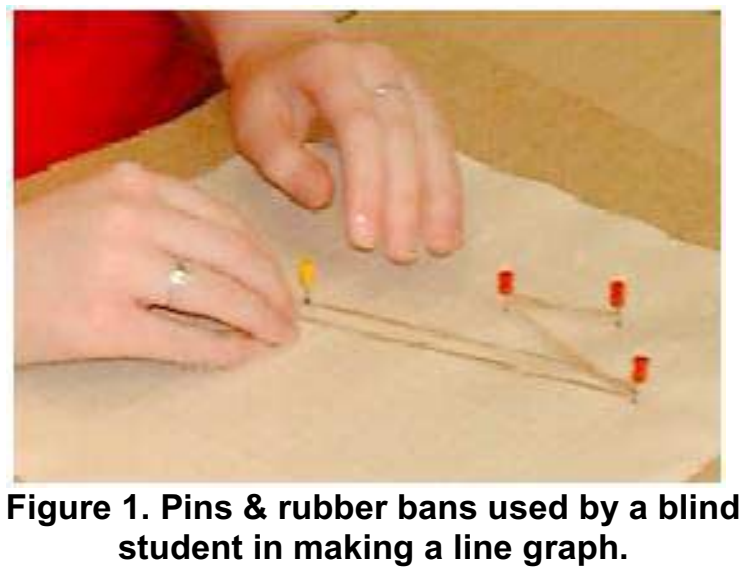

To address these problems and provide blind students with a better way to create and manipulate graphs, we use a computer based approach, in which a low-cost haptic device and the Internet are used. We have chosen the Logitech WingMan Force Feedback (FF) Mouse (Figure 2 ), as the main user interface. Most of the VR-based assistive tools use expensive devices so that it is still far away for blind people to actually benefit from the research findings. Therefore, the affordable price of the WingMan FF mouse and its force feedback capability makes it the ideal tool for this work. Although it has several limitations such as the small workspace, weak force feedback and only provides two degrees-offreedom, it is still capable of presenting graphs which are basically in 2D nature. Moreover, our previous study has shown that users' performance on the WingMan FF mouse can be significantly improved by adding audio feedback [4].

Our tool is Web-oriented and available on our Web site [5]. Users do not need to download the program, instead they can simply visit the website and use the tool directly. All users need in order to experience the haptic feedback is the WingMan FF mouse and the Immersion TouchSense Web plug-ins (which are freely available). The tool is a Java Applet which is embedded in JavaScript and HTML. Using the haptic technology on the Web allows us to take advantages of the Internet for our blind users. The Internet has particular benefits to blind people, for example, they can reach out for information without leaving home. Their communication and manipulation capabilities can also be extended through the Internet. Therefore, by integrating the low-cost haptic device with the Internet makes the tool more versatile and truly usable to blind people.

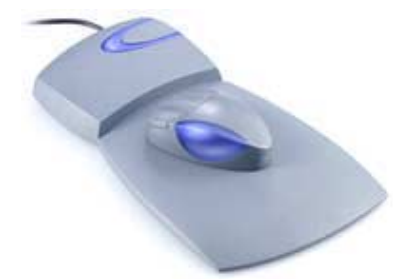

Figure 2. Logitech WingMan Force Feedback
mouse.

\section{Web-based Graph Generating Tool}

The tool is represented as Web pages which can be accessed using a standard Web browser with a Java virtual machine enabled. Maintenance of the tool is easy as modifications to the tool and updates of the tool features can be done on the server without the need to redistribute the program. Wise et al. have also used a Web-based haptic tool for blind students to access science education software [7].

Currently, our tool consists of two functional components: automatic graph generation and interactive drawing. The automatic graph generation works like the graph-plotting tool in Microsoft Excel that plots a graph based on the selected data. Users only need to input the data set into the tool and then the graph will be rendered on the computer screen. Blind users can explore the graph through the WingMan FF mouse with audio feedback. The rendered graphs can also be printed out and subsequently raised on the swell paper so that blind users can present them to sighted or blind colleagues for communication purpose.

The interactive drawing provides an opportunity for blind users to draw graphs manually. It is particularly useful in the classroom environment where pins and rubber bands can be replaced by a safer and more convenient tool that is linked to a computer. so that graphs can be saved. With this kind of tool, we hope that blind students can learn to plot graphs more easily and have a deeper understanding of how a graph is made and how they are used to present information. A virtual grid is presented on the computer screen on which blind users can use either the keyboard or the WingMan FF mouse to navigate. By pressing keys or mouse buttons, users can define the places on the grid where they would like to 
draw. The finished graph again can be printed out or just explored through the WingMan FF mouse with multimodal representations.

\subsection{Automatic Graph Generation}

This part of the tool can handle three types of graphs: line graphs, bar charts, and pie charts. A simple tree structure directory is used to manage different types of graphs. A main page lists the hyperlinks to the three types of graphs. The general arrangement of the tool and a line graph sample are shown in Figure 3. It can be divided into three parts: graph display area, data entry field, and control buttons group. The graph display area occupies most of the screen and is located above the other two areas. At the present stage, 10 data entry boxes are available in the data entry field but more will be provided in the future. There are two buttons on the right of the data entry field. One is the ' $\mathrm{OK}$ ' button which renders the graphs based on the data in the data entry fields. The other one is the 'Random' button which generates a set of randomised numbers for quick demonstration.

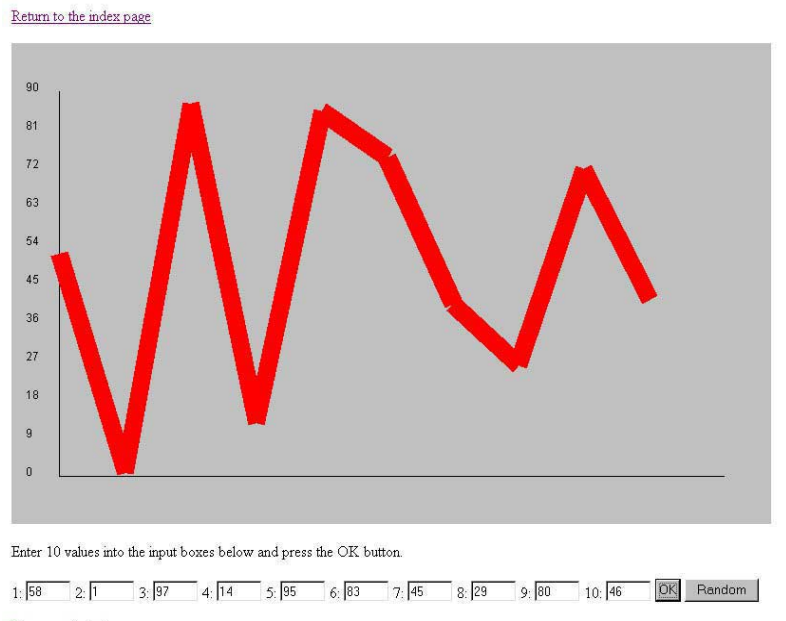

Return to the index page

Figure 3. Automatic graph generation tool.

To plot a graph using this tool, blind users would navigate on the interface to change the cursor focus on the data entry field by pressing the 'Tab' key (A standard screen reader is required for the navigation purpose). After entering the data, they would change the focus to the 'OK' button and then press 'Enter' key. The graph will be rendered onto the screen and the users would be able to use the WingMan FF mouse to extract information based on the haptic and audio feedback.

\subsection{Interactive Drawing}

The interactive drawing part of the tool is still under development however it currently provides functionality for users to perform line drawings. The interface is displayed in Figure 4. It has a simple layout which consists of a grid with 14 rows and 25 columns. It is very similar to graph paper that a sighted person would use. Drawing is done in the grid area and maximum of two lines can be drawn on the same graph.

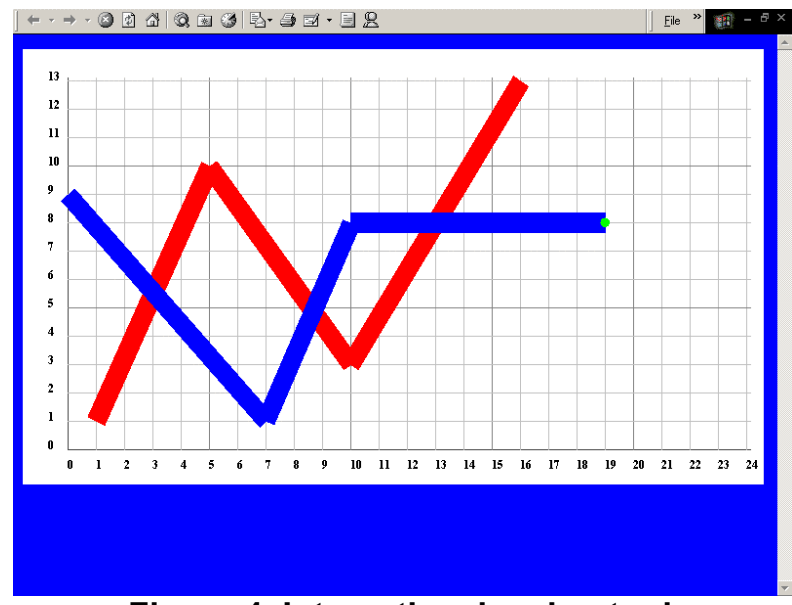

Figure 4. Interactive drawing tool.

To draw a line on the grid, two input methods are available: keyboard and WingMan FF mouse. In the keyboard mode, blind users rely on the audio information to determine the cursor location on the grid. In the mouse mode, they can use their sense of touch to count the number of rows and columns based on the force feedback from the mouse. Audio information is also available in the mouse mode. The audio consists of speech and nonspeech sounds. The speech is pre-recorded messages in Wave file format and it is mainly used to read out the coordinates of the cursor. The non-speech sound is generated using Java MIDI and it is used to give audio confirmation of mouse rolling over a major gridline or when a point on the grid is selected for drawing a line.

In the initial design, to draw a line in the mouse mode the user needs to double click on a grid intersection in order to start the line. Afterwards, users would single click on the next point on the grid to form a line segment. At the end point of the line, users must double click again to indicate that is the last point on the line. In the keyboard mode, several keys are assigned (Table 1).

\section{Table 1 Key assignment.}

\begin{tabular}{|l|l|}
\hline Key & Function \\
\hline Enter & As 'double click' on the mouse \\
\hline 5 on the number pad & As 'single click' \\
\hline Arrow keys & For moving the cursor \\
\hline '-', minus key & To delete the point that just drawn \\
\hline Ctrl + Delete & To erase all the drawings \\
\hline
\end{tabular}


Once the graph is drawn, users can explore it by using the WingMan FF mouse; they can check whether they have made mistakes on the drawing or simply trace the line to find out the trend of data. Alternatively, users can print out the graph and raise it on swell paper and then explore it in the traditional way. In either case, the system provides a tool that not only allows blind people to draw graph on their own, but also provide means that blind people can use to explore what they have drawn.

\section{Implementation}

To implement the drawing tool, we use combination of JavaScript and Java Applets, the Immersion TouchSense Java SDK for the haptic effects, Java MIDI for the nonspeech sound and pre-recorded voice for speech output. The key issue here is how to implement the haptic effects to represent different types of graphs. The two major force effects provided in the Immersion TouchSense SDK are used to construct the haptic objects. They are the grid effect, and enclosure effects which include elliptical and rectangular effects. The grid effect is used to represent the drawing grid on the interactive drawing tool interface while the enclosure effect is used to assemble different types of graphs. Enclosure effects are defined as areas bound by force walls. The mouse cursor can be trapped inside the areas unless the user applies larger force to overcome the restraining force.

\subsection{Line Graph Implementation}

As lines are comprised of segments and each segment can be regarded as a thin rectangular section, a rectangular enclosure effect is used. A rectangular enclosure effect is surrounded by four walls: top, bottom, left, and right. Each wall has outer and inner surfaces. Each wall and surface can be enabled or disabled separately. Therefore, different effects can be simulated by a combination of enabled and disabled components. For example, a ridge can be formed by only enabling the outer surfaces of the four walls, whereas, a groove is formed by enabling the inner surfaces of the walls.

To simulate a line segment, we only enable the top and bottom walls of the rectangular enclosure effect and keep a very small gap in between. Moreover, only the inner surfaces of these two walls are used so that a groove is formed to trap the mouse cursor. To represent the different trends on a line, the enclosure effect is rotated to the desirable angle (Figure 5). There is a limitation in the TouchSense plugin on the number of enclosure effects that can be rendered simultaneously. We can only have 20 enclosure effects showing so that each line can only have 10 segments for a two-line graph. This limitation can be overcome by using other modelling techniques such as creating a magnetic field around the line. At the current stage, we use the enclosure effect for its simple implementation and compatibility with multi-line graphs.

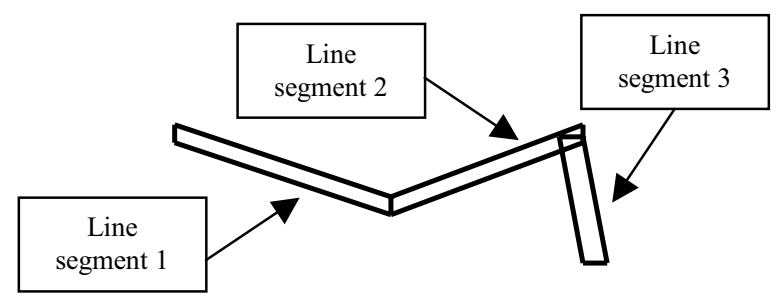

Figure 5. Arrangement of enclosure effect to simulate a line.

The audio is implemented using Java MIDI. A piano note is played continuously and varies in pitch according to the mouse cursor position. High data value points are mapped to high pitched notes and vice versa [6]. Therefore, by moving the mouse along the line, various pitches will play and inform users about the trend of the data. The sound is only played when the mouse cursor falls into the bounded area.

\subsection{Bar Chart Implementation}

Again we use the rectangular enclosure effect to model the bar charts due to the shape of the bars. All the bars are located on the $\mathrm{x}$ axis and without a gap in between. This arrangement is different from the traditional tactile diagrams on which bars are usually placed with a small gap $(\sim 3 \mathrm{~mm})$ in between. The gaps are designed for easy distinction between neighbouring bars through tactile sensation. However, only kinaesthetic sense is available on the force feedback mouse, the role of gaps between bars become less important. This has been proven by our previous study in which users evaluated two different types of bar settings. The users' performance was slightly better on the bars without gaps [7]. Although the differences are not significant, comments from the users showed their preference towards the closely placed bars.

The enclosure effects are defined so that all four walls and the outer and inner surfaces are enabled, so that users can touch both the inside and outside of the bar from all directions. This enables users to explore the bars and make comparisons either from the inside or around the outside. A discrete sound is used to present the bar value. The same pitch-to-value mapping is used again; the higher the bar, higher the pitch. The sound is triggered when the mouse cursor enters a bar.

\subsection{Pie Chart Implementation}

To model a pie chart, both elliptical and rectangular enclosure effects are used. The elliptical effect is used for the pie circumference whereas the rectangular effect is 
used for the dividing borders on the pie. Figure 6 shows how these two effects are combined to assemble a pie chart. The elliptical enclosure effect does not have four walls and instead it has only the outer and inner rings. There are also outer and inner surfaces of each ring. In the initial design, to simulate the pie circumference, only the inner surface of the inner ring was enabled. Therefore, users would only feel the inside area of the pie. After an evaluation study [8], we found that users preferred to move along the edge of the pie to check the size of each portion. In order to make this process easier, we modified the settings of the elliptical enclosure effect so that not only the inner surface of the inner ring is enabled but also its outer surface as well as the inner surface of the outer ring. Therefore, users would be able to feel the magnetic force on the pie edge and then trace along it.

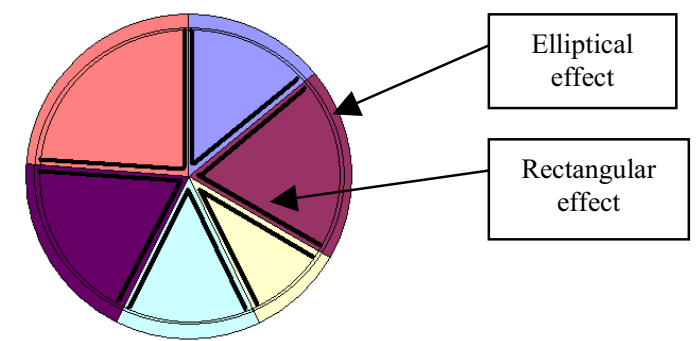

\section{Figure 6. Arrangements of enclosure effects to form a pie chart.}

The pie is divided into several portions in order to represent the different percentages of the value distribution. To model the dividing border, a rectangular enclosure effect is used in the same way as in the line graph. The rectangular effects are orientated at the centre of the pie and pointing out to the edges. A discrete sound mapping is used again. The pitch is mapped to the proportion of the pie division. The same triggering mechanism is used to play the sound.

\subsection{Drawing Grid Implementation}

The haptic grid provides blind users with information that they can rely on to draw lines. Implementation of the grid is not very difficult as a grid effect is provided in the Immersion TouseSense SDK. However, it is not easy to provide a grid with suitable force feedback to every user. The size of the grid, number of gridlines and distance between gridlines affect the resolution of the graph and the distribution of force on each gridline. Gridlines are modelled as ridges so that whenever the force feedback mouse rolls over a gridline, users would feel the 'clicks' from the mouse. Based on this information, users can count on how many rows or columns they have crossed.

The same haptic modelling of line segments that is described earlier is used in the drawing tool. The audio part of the drawing tool consists of speech and non-speech sounds. The speech is mainly used to tell users about their cursor position on the grid and other useful information such as the number of points that have been drawn. These files are played back when users pressed the associated keys or mouse buttons. The non-speech sound is used to present the interaction between the mouse and the gridlines as well as the trend of the data line. The fifth and the multiples of fifth gridlines (i.e. $5^{\text {th }}, 10^{\text {th }}, 15^{\text {th }}$, etc) are considered as the major lines so that whenever mouse cursor roll-over those lines, a MIDI sound will be played. This acts as an informative cue to the users who can count their position on the grid. The representation of the audio on the data lines are the same as the representation used on the other line graphs.

\section{Evaluations}

The evaluations of the graph generator tool have been done in two parts: experiments with sighted people and with blind people. The aim of the evaluation is to find out how well the users can use the tool to generate graphs as well as explore them to receive useful information. The experiments with sighted people were designed to evaluate the effectiveness of the multimodal representation (haptic and auditory) of various graphs. Sighted people were blind-folded during the experiment. As with our previous study, there were no major differences between blind people and blind-folded sighted people's performance on the multimodal graphs [9].

In order to evaluate the usability of the graph generator tools, we have been conducting case studies with blind people. The case studies use the Think Aloud approach in which users provide verbal feedback while they are using the experimental platform. It is more of a qualitative approach than a quantitative one as users directly point out the good and bad points of the tools. This is particularly useful in the design process as any drawbacks that are unforeseen in the design stage can be identified and corrected. There were three major issues investigated in the case studies: navigation in the interface, using the tools to create graphs, and extracting information from the created graphs.

\subsection{Evaluations of Automatic Graph Generation}

The evaluation consisted of a formal experiment with sighted people and two case studies with blind people. In the formal experiment, a between-group design was used. Eighteen people were recruited and evenly divided into three groups. Each group performed a set of tasks on the pie charts in one of three experimental conditions: Audio only, Haptics only, and Audio with Haptics [8]. They were asked to (1) locate the largest \& smallest divisions, (2) locate the two most similar divisions in value. There 
were sixteen graphs in each condition and a three-minute time limit was placed on each graph. The answer accuracy, task completion time and subjective workload were measured to assess users' performance.

A summary of the experimental results is given in Figure 7. An ANOVA and Post Hoc Tukey's HSD tests were performed and showed that there was a significant difference between the accuracy results in three conditions. The Audio with Haptics condition had a high percentage accuracy (79\%) and Haptic only condition achieved the lowest accuracy (10\%). Moreover, users took longer time in Haptics only condition (100 sec.) and much less in the Audio with Haptics condition (59 sec.). The statistical analysis revealed a significant difference between these two conditions. However, there was no significant difference between these two conditions and the Audio condition. The Haptic only condition was also perceived as the most difficult condition while Audio with Haptics was the easiest. The statistical analysis showed that there was a significant difference between all conditions.

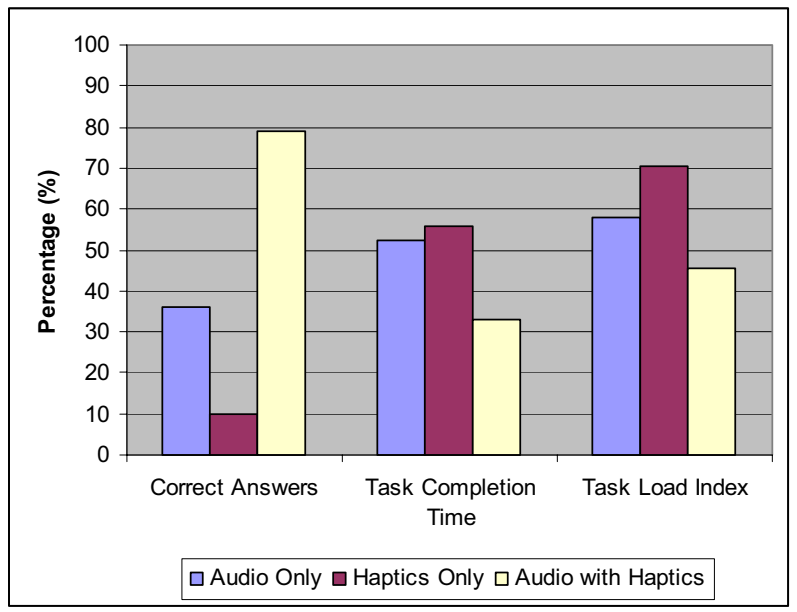

Figure 7. Summary of the experiment results.

In the case studies, two blind people evaluated the tool of automatic graph generation. They were asked to use the tool to create some graphs based on a set of provided data and then they performed the same task as sighted people did in the formal experiment. Their performance was recorded as well as their comments about the tool.

Their comments can be summarised into following points:

- Navigation on the tool is easy;

- No problem with data entry;

- Haptic and audio representations are informative;

- Force on the pie edge is not strong enough;

- Need to be able to turn the audio on and off;

- Need practice to use the mouse successfully.

\subsection{Evaluations of Interactive Drawing}

The evaluation consisted of a pilot study with sighed people and a case study with a blind person. In the pilot study, six participants were divided into two groups and each group carried out the drawing task in both mouse and keyboard modes. One group did the task in the mouse mode first and then the keyboard mode. The other group did it in the reverse order. Participants were requested to draw two graphs (each with two lines) in each mode. The error rate, time to complete the drawing and workload index [12] were noted.

The results revealed several drawbacks of the interface design and hardware limitations. There were not many differences between the errors in the two different modes. Four kinds of errors were found including, extra points in the lines, points at the wrong place, missing points, and unrecognised double clicks. In terms of task completion time, participants spent less time in the keyboard mode (average $124 \mathrm{sec}$. compared to $240 \mathrm{sec}$. in the mouse mode). They also rated less workload demands in the keyboard mode (average 3.79 compared to 5.75 in the mouse mode).

Although the participants' error rates in both input modes are similar, they preferred using the keyboard to draw graphs. This is because the keyboard is more accurate in positioning the cursor and participants required less audio confirmation from the interface. As a result, the time spent to draw the graphs in the keyboard mode is also shorter.

Some observations during the experiments were noted as well as the comments from the participants. Some complaints were made about the forces on the gridlines. Some participants thought it was too strong while the others thought the opposite. Suggestions were also made about attaching audio feedback on the major gridlines in the keyboard mode so that users can count the movement of the cursor more efficiently.

It was noted that the force placed on the boundary of the drawing area was not strong enough to keep the cursor inside. This could cause confusion when the user is outside of the drawing area without noticing it and still trying to draw a point on the graph.

A major problem noticed in the experiment was that some participants had difficulties starting a line by double clicking. The program did not recognise the double click made so that no line was drawn. The audio associated with the starting of a line was also not informative enough to let user recognise a line has been started (or not).

Some participants suggested a function that would provide audio information about all the points that have been drawn so that they can confirm the results. Moreover, better audio feedback was required to assure them that a point had just been drawn. 
Based on the problems revealed in the pilot study some improvements were made to the system. They are:

- Adding speech read-out of the last point that users have drawn;

- Adding a speech read-out of all the points that users have drawn;

- To start a line, users only need to do one click instead of a double click;

- Improved audio feedback about the end point of the lines.

In the case study, a blind user performed the same task used in the pilot study on the improved interface. A Think Aloud was used to capture user's comments.

The user pointed out that the different sounds on the interface became a little annoying. He suggested that the gridline sounds could be turned off after the lines were drawn. A comment was received regarding the direction of the sound. Stereo panning of the sound would be useful to inform users about their cursor location on the graph.

The user also commented on the keyboard input mode. He thought the keyboard was a faster and more logical way to input information. It is more natural for him to input data using the keyboard. When drawing multiple lines, the user commented that an extra exploring mode might be required. The previously drawn line need not be displayed in the drawing mode so that there is no interference to users' drawing of a new line.

\section{Discussions}

The evaluations have confirmed the usefulness of the tools and their potential for blind people. With further improvements to the system, blind people's ways of creating and interacting with graphs could be improved.

Several issues were raised during the evaluation process. They can be classified into three categories: design of haptic features, design of the user interface, and influence from the force feedback device.

\subsection{Issues of Haptic Features Design}

In the design of haptic features, three factors have to be taken into account: force perception, and temporal and spatial occurrence of force. As revealed in the feedback of participants, individuals have different perception of force strength. The same amount of force can be perceived as weak by some participants but felt strong by others. This has made the design of haptic features on the interface more difficult, especially with gridlines. Therefore, care has to be taken in deciding the amount of force on the haptic objects. User trials during the design stage would be useful in obtaining a moderate force value. Alternatively, options can be provided to let a user to customise the force to fit their needs.
Temporal and spatial occurrence of the force effects are about when and where the force effects should occur. Based on the findings in the case studies with blind users, different modes should be provided to let users to draw and feel the graphs. The haptic features available during the drawing stage, i.e. gridlines, may not be necessary in the exploration stage. This matches the suggestions given by Edman on using gridlines on tactile diagrams [13]. Suitable selection of haptic features can help users to complete their tasks more effectively. Otherwise, useful features could become hindrances to users' work.

The purpose of the system is to provide haptic equivalence of visualisation. The placement and the formation of force effects determine whether blind users can successfully interpret the haptically rendered information. In our evaluation, we recognised that users would trace the edge of the pie in order to compare the size of different portions. Therefore, we placed a groove around the pie to assist users' exploration. The role of the pie circumference was not so obvious in the visual sense but it becomes more important in the haptic representation. Hence, we should consider this kind of difference when we are designing haptic equivalence of graphical information.

\subsection{Issues of User Interface Design}

In the design of the user interfaces containing haptic features, several factors have to be considered. First, the size of the interface (application window) on the computer screen has to be set carefully. In applications for blind people (especially the applications using the WingMan Force Feedback Mouse), the interface would be better to occupy the full screen so that the mouse cursor will not move out of the interface. Otherwise, users lose track of the cursor position and get confused.

Screen resolution is another important issue: The dimension of the force effects supported by the WingMan FF mouse is defined in screen coordinates. The resolution of the screen affects the size of the force effects as well as the interface size. Therefore, developers should check the users' screen resolution first before rendering the application window and the force effects.

As the application window can be resized and dragged to a new location, the program should be flexible enough to cope with these kinds of dynamic changes. So that after the application window is changed, the force effects are still matched with the graphical display.

Another major consideration issue in developing haptic assistive applications for blind people is the compatibility with screen reader software. There are several commonly used screen readers, e.g. JAWS, Supernova, WindowEyes, etc, which read out the information displayed on the screen. To change or activate some functions of these types of software, keys and combinations of keystrokes 
are used. It is important to check whether the key assignments of the haptic applications have conflicts with those used in the screen readers. Moreover, it is essential to run the screen readers on the application to check whether the information displayed can be interpreted.

\subsection{WingMan Force Feedback Mouse Influence}

This work is developed based on the WingMan FF mouse because of its low-cost and better software API support. However, the limitations of the device also affect the performance of the system:

- Only suitable for 2D representations;

- Very small workspace;

- Limited amount of force feedback;

- Confusing mouse rotation, without effect on the cursor position;

- Single point contact.

The major problem with the device is its lack of Operating System (OS) support. Logitech has discontinued this device so that it is not compatible with the latest OS such as Windows 2000 \& XP. This would not be a problem if majority of blind people are still using Windows 98 and Me. However, the system will not be future proof.

\section{Future Work}

Future work will focus on three different areas: looking for an alternative low-cost haptic device, incorporating more functionality into the system, and introducing synthesized speech. We hope that with more mature haptic technology and extended use of Internet, more and more applications will be developed to link these two technologies together to improve blind people's quality of life.

\section{Conclusions}

The delivery of haptic information via the Web to help blind people to create and manipulate graphs independently has been introduced in this paper. Evaluations of the system that we have developed have been conducted and the experimental results have shown the usefulness of the system in allowing blind people to create graphs in two different ways: automatically or interactively. Suggestions from the users' comments have been used to improve the system. Issues to be considered when designing effective haptic assistive tools for blind people have been discussed. With continuing improvements of haptic technology both in hardware and software, more applications can be identified and developed for people with special needs. As a result, their quality of life will be improved significantly.

\section{Acknowledgements}

This research work is funded by EPSRC Grant GR/M44866, ONCE (Spain) and Virtual Presence Ltd.

\section{References}

[1] Yu W., Brewster S.A., Multimodal Virtual Reality Versus Printed Medium in Visualization for Blind People. Proceedings of The Fifth International ACM Conference on Assistive Technologies, 2002: p. 57-64.

[2] Kamel H. M., Landay J.A., Sketching Images Eyes-free: A Grid-based Dynamic Drawing Tool for the Blind. Proceedings of The Fifth International ACM Conference on Assistive Technologies, 2002: p. 33-40.

[3] Van Scoy F., Kawai T., Darrah M. \& Rash C., Haptic display of mathematical functions for teaching mathematics to students with vision disabilities: design and proof of concept. Haptic Human-Computer Interaction. Springer LNCS, Vol 2058. 2000. pp 31-40

[4] Dimigen G., Scott F., Thackeray F., Pimm M., Roy A. W. N., Career expectations of Brithish visually impaired students who are of school-leaving age. Journal of Visual Impairment and Blindness, 1993(87): p. 209-210.

[5] Yu W., Brewster S.A., Comparing Two Haptic Interfaces for Multimodal Graph Rendering. Proceedings of the 10th Symposium on Haptic Interfaces for Virtual Environment and Teleoperator Systems (Haptics 2002), (Florida, USA). IEEE, pp. 3-9.

[6] Multivis project website, http://www.multivis.org.

[7] Wies E. F., Gardner J. A., Sile O'Modhrain M., Hasser C. J., Bulatov V. L., Web-based Touch Display for Accessible Science Education. Haptic Human-Computer Interaction, 2001. LNCS 2058: p. 52-60.

[8] Mansur D.L., Blattner M., Joy K., Sound-Graphs: A numerical data analysis method for the blind. Journal of Medical Systems, 1985. 9: p. 163-174.

[9] Yu W., Reid D., Brewster S. A., Web-Based Multimodal Graphs for Visually Impaired People. Proceedings of The First Cambridge Workshop on Universal Access and Assistive Technology (CWUAAT), 2002: p. 97-108.

[10] Yu W., Cheung K., Brewster S.A., Automatic Online Haptic Graph Construction. Proceedings of EuroHaptics 2002, 2002: p. 128-133.

[11] Yu W., Ramloll R., Brewster S., Ridel B., Exploring Computer-Generated Line Graphs through Virtual Touch. Proceedings of The Sixth International Symposium on Signal Processing and Its Applications, 2001. 1: p. 72-75.

[12] Hart S. G. and Wicken S. C., "Workload assessment and predication", in MANPRINT, an approach to systems integration. 1990, Van Nostrand Reinhold: New York.

[13] Edman, P.K., Tactile graphics. 1991: American Foundation for the Blind. 